\title{
Chiral lattice fermions from staggered fields
}

\author{
Simon Catteralle \\ Department of Physics, Syracuse University, Syracuse, New York 13244, USA
}

(Received 24 January 2021; accepted 9 June 2021; published 6 July 2021)

\begin{abstract}
We describe a proposal for constructing a lattice theory that we argue may be capable of yielding free Weyl fermions in the continuum limit. The model employs reduced staggered fermions and uses site parity dependent Yukawa interactions of Fidkowski-Kitaev type to gap a subset of the lattice fermions without breaking symmetries. The possibility for such symmetric mass generation is tied to the cancellation of certain discrete anomalies arising in the continuum limit. The latter place strong constraints on the number of lattice fermions - constraints that are satisfied by this model. We present numerical results for the model in two dimensions which support this scenario.
\end{abstract}

DOI: 10.1103/PhysRevD.104.014503

\section{INTRODUCTION}

It has long been a goal of lattice field theory to be able to describe continuum chiral gauge theories. All of the standard local lattice fermion prescriptions; Wilson, staggered, domain wall and overlap appear only capable of describing vectorlike theories. The central reason for this is well known - the Nielsen-Ninomiya theorem asserts that a wide class of fermion discretizations with exact chiral symmetry will necessarily contain equal numbers of left and right-handed fields [1].

Two paths have typically been followed to try and evade this theorem. In domain wall approaches one starts from a lattice in five dimensions and then introduces a domain wall in the extra dimension which binds one fermion of fixed four dimensional chirality. On a finite lattice one must necessarily introduce an antidomain wall binding a fermion of opposite chirality. Unfortunately in the presence of a fluctuating gauge field these modes become coupled once more and the continuum limit describes Dirac fermions again. Recently Grabowska and Kaplan proposed a modification of the domain wall prescription that used a deformation of the fermion kernel to decrease the coupling to the mirror fermions located on the antidomain wall [2]. However, it is unclear whether this proposal will be sufficient at the nonperturbative level.

A second way to proceed is to start with a vectorlike theory directly in four dimensions and attempt to give cutoff scale masses to states of one chirality using strong multifermion interactions. Perhaps the first example of such

Published by the American Physical Society under the terms of the Creative Commons Attribution 4.0 International license. Further distribution of this work must maintain attribution to the author(s) and the published article's title, journal citation, and DOI. Funded by SCOAP ${ }^{3}$. a mirror model was given by Eichten and Preskill [3]. The early numerical work to test this idea made use of Wilson and staggered lattice fermions [4-8] and appeared to invalidate the approach - to generate large mirror masses required large four fermion or Yukawa couplings and typically this resulted in the formation of symmetry breaking condensates coupling left and right-handed states via Dirac mass terms [9].

More recently this approach was revived for lattice fermion actions with superior chiral properties-in a series of papers Poppitz et al. have investigated models using overlap fermions [10-12] while a gauge invariant path integral measure for overlap chiral fermions in $S O(10)$ was constructed in [13]. An earlier proposal combining domain wall fermions and appropriate four fermion interactions was made by Creutz et al. in [14]. However, again, the overall conclusion of these studies was that it was difficult, if not impossible, to decouple the chiralities in the continuum limit. ${ }^{1}$

However, in recent years, a series of developments in condensed matter physics have provided new insights into the problem. One of the key new ingredients has been the discovery of models capable of symmetric mass generation. This field was launched by the seminal paper of Kitaev and Fidkowski (FK) [16] who showed that it was possible to design a four fermion interaction that was capable of generating masses for precisely eight one dimensional Majorana modes without generating symmetry breaking fermion condensates. Subsequent work generalized this to higher dimensions finding that sixteen Majorana fermions are needed in three and four (spacetime) dimensions [17-20].

\footnotetext{
${ }^{1}$ A possible exception to this was Lüscher's formal construction of a path integral for $U(1)$ chiral gauge theory in [15].
} 
It is now understood that the appearance of specific numbers of fermions in theories where symmetric mass generation is possible, is tied to the cancellation of certain discrete anomalies $[21,22]$. Indeed, one way to understand the observation of symmetry broken phases in some of the earlier work on lattice four fermion theories is that they arise as a consequence of the failure to cancel off these discrete anomalies. To replicate a nonzero anomaly in the UV requires massless states in the IR which can arise as Goldstone modes arising after the spontaneous breaking of symmetries via fermion condensates.

The phenomenon of symmetric mass generation has been seen in recent numerical studies of vectorlike lattice models in dimensions from two to four [23-30]. While massive symmetric phases had been observed in early lattice studies of Higgs-Yukawa theories, they were typically separated from the weak coupling regime by regions where lattice symmetries were spontaneously broken by the formation of bilinear condensates and the massive symmetric phases were interpreted as lattice artifacts [4-8]. In the more recent work these massive symmetric phases in theories can be directly connected to the massless phase via a continuous phase transition thereby allowing for a continuum limit. One crucial difference between the new work and these older studies is the use of reduced staggered fermions which carry half the number of degrees of freedom of regular staggered fermions and can be thought of as lattice analogs of continuum Majorana fermions. They will form a key ingredient in this proposal for constructing chiral lattice theories.

It was realized some years ago by $\mathrm{Xu}$, Wen, and others in the condensed matter community that symmetric mass generation might allow one to construct anomaly free chiral lattice gauge theories and several proposals have been made [19,31-33]. However, these models use Hamiltonian formulations and continuum topological arguments to make the case for symmetric mass generation. The construction in this paper aims to furnish an explicit Euclidean lattice path integral in which single component relativistic lattice fermions can be gapped by a FK type interaction thereby producing a low energy theory which can be shown to produce chiral fermions in the continuum limit.

We start our discussion with a quick review of reduced staggered fermions and how they may be given a mass without breaking symmetries using a four fermion interaction. However, this construction yields a continuum limit describing massive Dirac fermions. To target a chiral theory requires different field content and interactions. We argue that the requirement that the lattice model yield the correct number of massless Majorana fermions in the continuum limit suggests a specific field content and an interaction of Fidkowski-Kitaev type.

We then discuss numerical results from simulations of the simplest model in two dimensions and provide evidence that indeed the interactions we propose are capable of decoupling the relevant lattice modes without the formation of bilinear condensates. We summarize our conclusions and discuss open questions in the final section of the paper.

\section{REDUCED STAGGERED FERMIONS AND SYMMETRIC MASS GENERATION}

The usual staggered fermion action in $D$ dimensions is easily arrived at by spin diagonalizing the naive fermion action on a hypercubic lattice and takes the form [34]

$$
S=\sum_{x, \mu} \eta_{\mu}(x) \bar{\chi}(x) D_{\mu}^{S} \chi(x)+\sum_{x} m \bar{\chi}(x) \chi(x)
$$

where $\eta_{\mu}(x)=(-1)^{\sum_{i=0}^{\mu-1} x_{i}}$ are the usual staggered fermion phases and the symmetric difference is given by

$$
D_{\mu}^{S} \chi^{a}(x)=\frac{1}{2}\left(\chi^{a}(x+\mu)-\chi^{a}(x-\mu)\right) .
$$

If $m=0$ a further reduction is possible by keeping only one (single component) fermion at each lattice site. Explicitly we introduce the projectors $P_{ \pm}$defined by

$$
P_{ \pm}=\frac{1}{2}(1 \pm \epsilon(x))
$$

where the site parity is given by $\epsilon(x)=(-1)^{\sum_{\mu=0}^{D-1} x_{\mu}}$. The lattice action decomposes into

$$
S=\sum_{x, \mu} \eta_{\mu}(x)\left(\bar{\chi}_{+}(x) D_{\mu}^{S} \chi_{-}(x)+\bar{\chi}_{-}(x) D_{\mu}^{S} \chi_{+}(x)\right)
$$

where $P_{+} \chi=\chi_{+}$etc. The reduction we need corresponds to, for example, retaining only the fields $P_{+} \chi$ and $P_{-} \bar{\chi}$. This results in the reduced staggered fermion action whose continuum limit corresponds to $2^{D / 2-1}$ Dirac fermions or equivalently $2^{D / 2}$ Majorana fermions [35].

$$
S=\sum_{x, \mu} \chi^{a}(x) \eta_{\mu}(x) D_{\mu}^{S} \chi^{a}(x)
$$

where we have relabeled $\bar{\chi} \rightarrow \chi$ on odd parity lattice sites. This action is invariant under both discrete rotations and a staggered shift symmetry:

$$
\chi(x) \rightarrow \xi_{\mu}(x) \chi(x+\mu)
$$

where $\xi_{\mu}=(-1)^{\sum_{i=\mu+1}^{D} x_{i}}$ [35]. The reduced staggered action is also invariant under a $U(1)$ symmetry

$$
\chi(x) \rightarrow e^{i \alpha \epsilon(x)} \chi(x)
$$

While the single flavor theory does not allow for a mass term this can be remedied by introducing multiple flavors 
of reduced staggered field. To avoid fermion bilinear terms the simplest model requires four reduced staggered fields transforming under an $S U(4)$ global symmetry

$$
\begin{aligned}
S= & \sum_{x, \mu} \chi^{a}(x) \eta_{\mu}(x) D_{\mu}^{S} \chi^{a}(x) \\
& -\frac{G^{2}}{8} \sum_{x} \epsilon_{a b c d} \chi^{a}(x) \chi^{b}(x) \chi^{c}(x) \chi^{d}(x) .
\end{aligned}
$$

Notice that the four fermion terms break the $U(1)$ symmetry down to $Z_{4}$ which acts on the lattice fermions as

$$
\chi(x) \rightarrow i \epsilon(x) \chi(x)
$$

This combination of shift, $Z_{4}$ and $S U(4)$ symmetries forbid any fermion bilinear operator from appearing in the quantum effective action. One intuitive way to understand how a fermion mass can arise in these models is to rewrite the four fermion operator as

$$
\epsilon_{a b c d} \chi^{a}(x) \chi^{b}(x) \chi^{c}(x) \chi^{d}(x)=\Omega_{a}(x) \chi^{a}(x)
$$

corresponding to a bilinear mass term formed by pairing an elementary fermion with a composite fermion $\Omega_{a}(x)=$ $\epsilon_{a b c d} \chi^{b}(x) \chi^{c}(x) \chi^{d}(x)$. It is easy to see that a condensate of this four fermion operator arises in the strong coupling limit $G \rightarrow \infty$ which can hence be interpreted as generating a fermion mass in that same limit. Since one expects massless fermions at $G=0$ there must be at least one phase transition separating the massless and massive symmetric regimes. $^{2}$

Evidence of a continuous transition separating these phases has been seen in both three and four dimensions [23-28]. To underline these conclusions we include a plot in Fig. 1 of the four fermion condensate in the four dimensional model taken from our earlier paper [27]. The rapid increase of the condensate close to $G \sim 1$ is indicative of such a phase transition which is borne out by further analysis as described in [27]. In this plot the coupling $\kappa$ that appears is the coefficient of an additional scalar kinetic term which must be tuned in the four dimensional theory to see this direct transition between massless and massive symmetric phases. The appearance of this additional operator is natural within a RG framework since the scalar kinetic first becomes marginally relevant in four dimensions.

These results encourage the belief that it is possible to achieve symmetric mass generation in relativistic lattice theories based on reduced staggered fermions even in four dimensions. However, since reduced staggered fields yield Dirac fermions in the continuum limit the mechanism

\footnotetext{
${ }^{2}$ The exception to this is two dimensions where the four fermion coupling is asymptotically free and one sees [29] a single symmetric gapped phase for all nonzero lattice couplings.
}

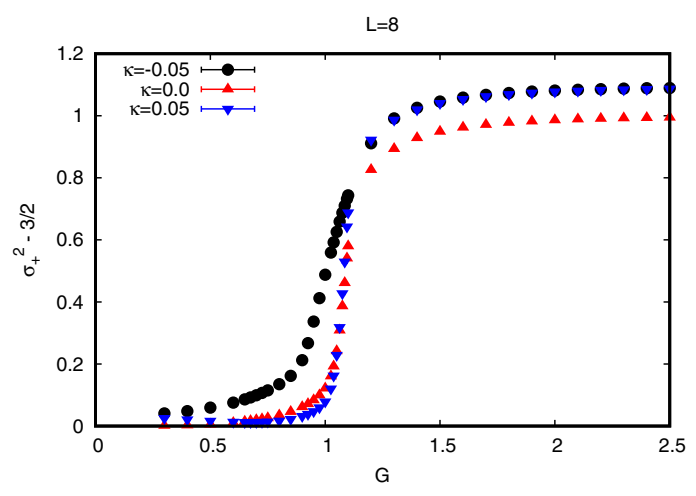

FIG. 1. Four fermion condensate vs $G$.

described above is only capable of generating mass in vector-like theories. To target a chiral theory we need to modify the quartic interactions in a manner that subjects only a subset of the reduced staggered field to quartic interactions. In a staggered theory there is a natural way to do this by dividing the lattice field into its even and odd site parity components. One is thus led to consider models in which Yukawa interactions are applied only to say even parity fields. We discuss the necessary structure for these interactions in the next section.

\section{MODIFIED QUARTIC INTERACTIONS}

We consider an action of the form:

$$
\begin{aligned}
S= & \sum_{x, \mu} \eta_{\mu}(x) \chi^{a}(x) D_{\mu}^{S} \chi^{a}(x) \\
& -\sum_{x}\left(G P_{+}+g P_{-}\right)\left[\chi^{T}(x) \Gamma_{A} \chi(x)\right]^{2}
\end{aligned}
$$

where $\Gamma_{A}$ are a set of antisymmetric matrices. As we will show in the next section the requirement that we target chiral fermions in the continuum limit requires the staggered fields to transform in a real representation of any underlying symmetry group. This will also guarantee the absence of additional perturbative and 't Hooft anomalies. One simple way to ensure this constraint it to look for real representations of a Euclidean rotation group. The smallest such group corresponds to $\operatorname{Spin}(7)$ which possesses a real, eight dimensional spinor representation. In this case the $\Gamma_{A}$ appearing in Eq. (11) are taken to be the pure imaginary, antisymmetric Dirac matrices appropriate for this representation. They are given by

$$
\Gamma_{A}=\left(\sigma^{123}, \sigma^{203}, \sigma^{323}, \sigma^{211}, \sigma^{021}, \sigma^{231}, \sigma^{002}\right)
$$

where the notation indicates that the Dirac matrices $\Gamma_{A}$ are built from tensor products of Pauli matrices. The resultant term is precisely the Fikdowski-Kitaev interaction which is known to be capable of generating mass for Majorana fermions in one dimension [16]. 
In practice we generate this term by coupling the bilinear $\chi^{T} \Gamma_{A} \chi$ to an auxiliary scalar $\sigma_{A}(x)$ with a simple Gaussian action $\sum_{x} \frac{1}{2} \sigma_{A}^{2}$. Notice that if these scalar fields are taken constant the Yukawa interaction just becomes a Majorana mass term for 8 real staggered fermions. In principle these eight real fermions can be organized into four complex staggered fields so that the theory will be invariant under the same $Z_{4}$ symmetry seen in the previous model targeting vectorlike theories. In the appendix we make another argument for the appearance of such a $Z_{4}$ symmetry based on a novel anomaly of free staggered fermions propagating on lattices with nontrivial topologies.

A crucial feature of the interaction we employ is that the associated Yukawa coupling depends on the parity of a lattice site. We will take $G$ to be large to drive symmetric mass generation in the even site parity sector while $g$ is kept small and serves merely to regulate a zero mode that appears in the odd parity sector at $g=0$ corresponding to the shift symmetry

$$
\chi_{-}(x) \rightarrow \chi_{-}(x)+\alpha_{-} .
$$

In the next section we will show that the remaining light fermions after gapping are capable of being organized in the continuum limit into 2 pairs of chiral fermions so that the final theory will contain sixteen Weyl fermions. This result is in accordance with the vanishing of a discrete spin$Z_{4}$ anomaly for systems of Weyl fermions corresponding to the transformations [20,22]:

$$
\begin{aligned}
& \psi_{L} \rightarrow-i \psi_{L} \\
& \psi_{R} \rightarrow+i \psi_{R}
\end{aligned}
$$

and takes the form

$$
\nu_{4}=n_{+}-n_{-} \bmod 16
$$

where $n_{ \pm}$denote the number of left and right-handed Weyl fields.

The Yukawa interaction we have described can be reduced to the subgroups $\operatorname{Spin}(N)$ for $2 \leq N<7$ by truncating the index $A$ to run from $1 \ldots N$ as described in [19]. The resultant theories with reduced symmetry still satisfy the anomaly cancellation condition and hence should still be capable of symmetric mass generation. Indeed, in the condensed matter literature the key feature which makes this mechanism possible is the nondegeneracy of the ground state of the FK interaction which continues to hold even in the reduced symmetry cases. Systems with a unique ground state cannot undergo spontaneous symmetry breaking and hence offer the possibility of symmetric mass generation.

\section{CHIRALITY AND THE CONTINUUM LIMIT}

In this section we discuss the continuum limit and show how the gapped theory can exhibit a chiral spectrum. As a warm up let us first consider the free two dimensional theory. We start by assembling the reduced staggered fields in a unit square of the lattice into a $2 \times 2$ matrix field $\Psi$ labeled by both spinor and flavor indices. If we employ the basis $\left\{I, \sigma_{1}, \sigma_{2}, \sigma_{1} \sigma_{2}\right\}$ it is given by

$\Psi=\left[\begin{array}{ll}\left(\chi_{+}(x)+i \chi_{+}(x+1+2)\right) & \left(\chi_{-}(x+1)+i \chi_{-}(x+2)\right) \\ \left(\chi_{-}(x+1)-i \chi_{-}(x+2)\right) & \left(\chi_{+}(x)-i \chi_{+}(x+1+2)\right)\end{array}\right]$

where the notation $x+1$ indicates the lattice site one step in the 1-direction from site $x$ etc. and we have suppressed the $\operatorname{Spin}(7)$ indices for simplicity. $\Psi$ is defined on a lattice with twice the lattice spacing and we added explicit \pm subscripts to $\chi$ indicating the site parity for clarity. Notice that this is a chiral basis for the two dimensional Dirac matrices and hence the upper row of this matrix contains right-handed fields while the left-handed fields are located in the lower row.

In addition, since we are employing real staggered fields the matrix field $\Psi$ satisfies a reality condition $\psi^{*}=\sigma_{1} \psi \sigma_{1}$ corresponding to the fact that it depends on only four real fields. This structure allows us to build two Majorana spinors from $\Psi$

$$
\begin{aligned}
& \Psi_{1}=\left(\begin{array}{l}
\chi_{-}(x+1)+i \chi_{-}(x+2) \\
\chi_{-}(x+1)-i \chi_{-}(x+2)
\end{array}\right) \\
& \Psi_{2}=\left(\begin{array}{l}
\chi_{+}(x)+i \chi_{+}(x+1+2) \\
\chi_{+}(x)-i \chi_{+}(x+1+2)
\end{array}\right) .
\end{aligned}
$$

Notice that each of these Majorana spinors depend only on lattice fields of fixed parity. If we are successful in generating a cutoff scale mass for the $\chi_{+}$modes we are left with a single light continuum Majorana fermion corresponding to $\Psi_{1}$. In the $g \rightarrow 0$ limit this becomes massless and is equivalent to a single left-handed Weyl fermion.

There is a similar story in four dimensions where we can build a $4 \times 4$ matrix field $\Psi$ from the staggered fields in a unit hypercube $[34,36] .^{3}$

$$
\Psi=\sum_{\left\{n_{\mu}=0,1\right\}} \chi(x+n) \gamma^{x+n}
$$

where $\gamma^{n_{\mu}}=\gamma_{0}^{n_{0}} \gamma_{1}^{n_{1}} \gamma_{2}^{n_{2}} \gamma_{3}^{n_{3}}$. In a chiral basis it is easy to see that $\Psi$ has the block structure

\footnotetext{
${ }^{3}$ In the lattice literature this is termed the spin-taste basis. It is equivalent to the Kähler-Dirac representation used in lattice susy constructions [37].
} 


$$
\Psi=\left(\begin{array}{ll}
E & O^{\prime} \\
O & E^{\prime}
\end{array}\right)
$$

where the $2 \times 2$ block matrices $E, E^{\prime}$ and $O, O^{\prime}$ contain only even and odd lattice site staggered fields. As in two dimensions the use of a real staggered field implies that $\Psi$ obeys a reality condition:

$$
\Psi=\gamma_{2} \Psi^{*} \gamma_{2}
$$

This in turn ensures that $O^{\prime}=-\sigma_{2} O^{*} \sigma_{2}$ and $E^{\prime}=\sigma_{2} E^{*} \sigma_{2}$. This condition ensures that the action can be written just in terms of the blocks $O$ and $E$ which depend only on the 16 real single component lattice fermions in an elementary hypercube.

Let us assume that we are successful in removing the even parity states from the low energy spectrum via the strong Yukawa interaction. If Lorentz invariance is restored in the continuum limit this would leave behind a pair of Majorana spinors determined only by the odd parity lattice fields:

$$
\Psi_{1}=\left(\begin{array}{c}
-\sigma_{2} O^{*} \sigma_{2} \\
O
\end{array}\right)
$$

Notice that a conventional Majorana spinor can be written

$$
\left(\begin{array}{c}
i \sigma_{2} \chi^{*} \\
\chi
\end{array}\right)
$$

The form of Eq. (23) thus suggests we adopt a generalized charge conjugation operation which acts both on the Lorentz and flavor indices of a pair of Weyl spinors. Again, if these Majorana spinors remain massless in the continuum limit they can be replaced by the pair of left Weyl fields contained in the block $O$.

Of course this analysis assumes we are able to give masses to the even parity fields while leaving the odd parity fields massless and noninteracting. We now give an argument in support of this conjecture. We start by rescaling the fields according to:

$$
\begin{aligned}
\chi_{+} & \rightarrow \frac{1}{G} \chi_{+} & \chi_{-} & \rightarrow \frac{1}{g} \chi_{-} \\
\sigma_{+}^{A} & \rightarrow \frac{1}{G} \sigma_{+}^{A} & \sigma_{-}^{A} & \rightarrow \frac{1}{g} \sigma_{-}^{A}
\end{aligned}
$$

The rescaled action (suppressing the Spin(7) indices) then reads:

$$
\begin{aligned}
S= & \frac{1}{x} \sum_{x}\left[\chi_{+} \eta_{\mu} D_{\mu} \chi_{-}+y \chi_{+} \Gamma_{A} \chi_{+} \sigma_{+}^{A}\right. \\
& \left.+\frac{1}{y} \chi_{-} \Gamma_{A} \chi_{-} \sigma_{-}^{A}+\frac{1}{2} y\left(\sigma_{+}^{A}\right)^{2}+\frac{1}{y}\left(\sigma_{-}^{A}\right)^{2}\right]
\end{aligned}
$$

where $x=G g$ and $y=\frac{g}{G}$. The equations of motion for the staggered fields are then

$$
\begin{aligned}
& \eta_{\mu} D_{\mu} \chi_{-}+y\left(\chi_{+} \Gamma_{A} \chi_{+}\right) \Gamma_{A} \chi_{+}=0 \\
& \eta_{\mu} D_{\mu} \chi_{+}+\frac{1}{y}\left(\chi_{-} \Gamma_{A} \chi_{-}\right) \Gamma_{A} \chi_{-}=0 .
\end{aligned}
$$

In the limit $y \rightarrow 0$ we see that the odd site field $\chi_{-}$, corresponding to the blocks $\left(O, O^{\prime}\right)$, is weakly coupled while the even parity field $\chi_{-}$, represented by $\left(E, E^{\prime}\right)$, is strongly coupled. In the next section we will provide numerical evidence that the fields in $\left(E, E^{\prime}\right)$ are in fact gapped and decoupled from the long wavelength modes given by the blocks $\left(O, O^{\prime}\right)$.

\section{EVIDENCE FOR SITE PARITY DEPENDENT MASS GENERATION}

In this section we present preliminary numerical evidence that the model is indeed capable of generating mass for the even site fermions without generating symmetry breaking fermion bilinear condensates. To avoid the need for tuning a scalar kinetic term results are presented only for two dimensions. ${ }^{4}$ However, both the structure of the FK interaction, the argument for symmetric mass generation, and appearance of chiral fermions in the continuum hold in both the two and four dimensional models.

We use a RHMC algorithm to simulate the system. For more details of our numerical methods see Appendix B. Measurements of the phase of the Pfaffian resulting from fermion integration show an absence of a sign problem for all the ensembles presented in this paper. Figure 2 shows a plot of the vevs of the odd and even four fermion operators as a function of the even site coupling $G$. The odd site coupling is fixed at $g=0.01$. We use lattices of size $L=8$ and $L=16$ and antiperiodic boundary conditions for the fermions in the (Euclidean) temporal direction. Notice that the two vevs differ by four orders of magnitude. We can extract expressions for these condensates from the fermion propagator which is given by inverting the fermion matrix $M$ which is given in odd/even block form by

$$
M=\left(\begin{array}{cc}
G \Sigma_{+} & \eta_{\mu} D_{\mu} \\
\eta_{\mu} D_{\mu} & g \Sigma_{-}
\end{array}\right)
$$

where $\Sigma_{ \pm}=\sigma_{ \pm}^{A} \Gamma_{A}$. This yields

$$
\langle\chi \chi\rangle=\left(\begin{array}{ll}
A & B \\
B & C
\end{array}\right)
$$

where

\footnotetext{
${ }^{4}$ Work in four dimensions is in progress and will be reported in a separate publication
} 


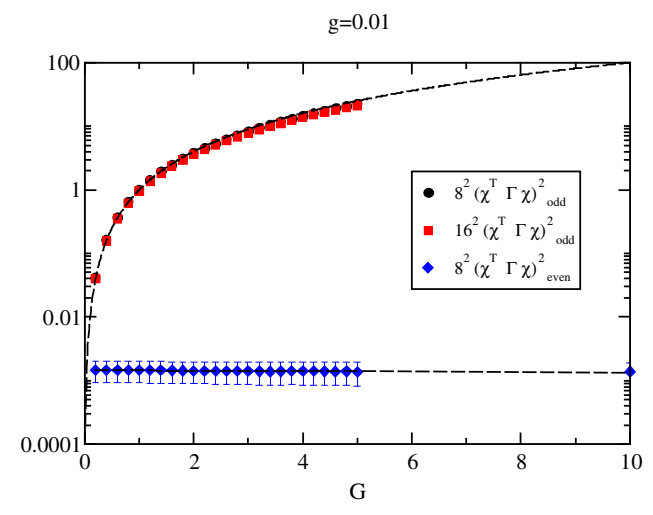

FIG. 2. $\left\langle\left(\chi^{T} \Gamma \chi\right)^{2}\right\rangle$ vs $G$ for $L=8,16$.

$$
\begin{aligned}
& \left\langle\chi_{+} \chi_{+}\right\rangle=A=g \Phi_{+} \\
& \left\langle\chi_{-} \chi_{-}\right\rangle=C=G \Phi_{-} \\
& \left\langle\chi_{+} \chi_{-}\right\rangle=B=\Sigma_{+}^{-1} \eta_{\mu} D_{\mu} \Phi_{-}
\end{aligned}
$$

where

$$
\Phi_{ \pm}=\left(x \Sigma_{ \pm}-\eta_{\mu} D_{\mu} \Sigma_{\mp}^{-1} \eta_{\mu} D_{\mu}\right)^{-1} .
$$

For $x \ll 1$ this yields

$$
\begin{aligned}
\left\langle\left(\chi_{+} \Gamma \chi_{+}\right)^{2}\right\rangle & =g^{2} \\
\left\langle\left(\chi_{-} \Gamma \chi_{-}\right)^{2}\right\rangle & =G^{2} .
\end{aligned}
$$

The dashed line shows a fit to the data assuming the coupling constant dependence shown in Eq. (33). In the same limit the off-diagonal propagator takes the free field form

$$
\left\langle\chi_{+} \chi_{-}\right\rangle=\frac{\eta_{\mu} D_{\mu}}{\Delta \mu^{2}}
$$

To gain more understanding of the implications of this asymmetric four fermion condensate it is useful to look at

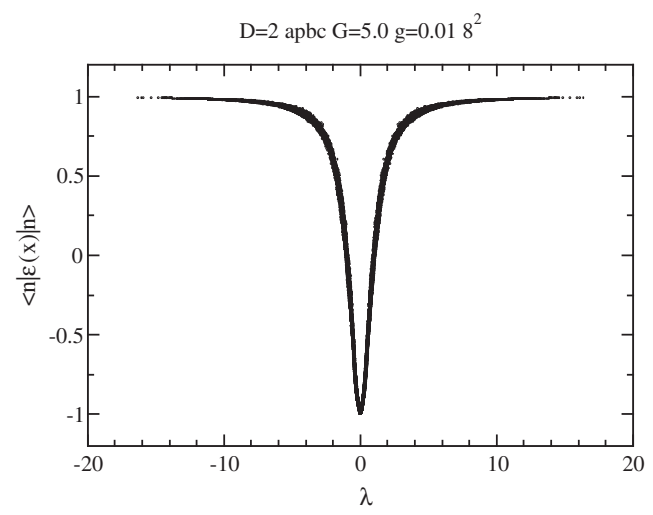

FIG. 3. $\langle n \epsilon(x) n\rangle$ vs $\lambda_{n}$ for $8^{2}$ lattice at $G=5.0$ and $g=0.01$.

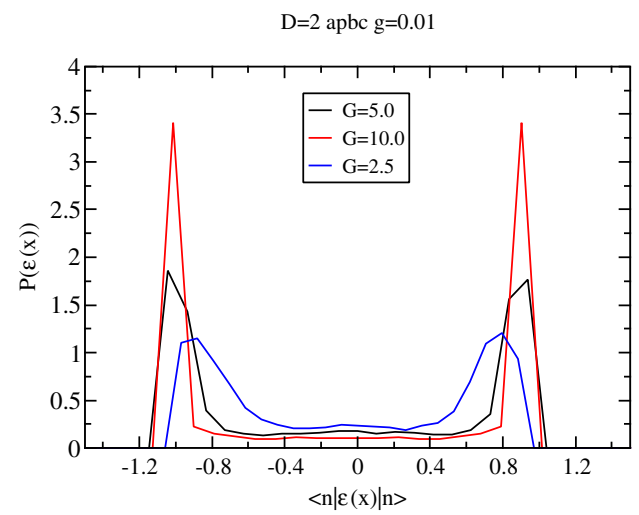

FIG. 4. Histogram of $\langle\epsilon(x)\rangle$ for $L=8$ and $G=5.0, g=0.01$.

the spectrum of the fermion operator. Figure 3 shows a plot of the matrix element of the site parity operator $\sum_{x} \phi_{n}^{2}(x) \epsilon(x)$ for each eigenstate $\phi_{n}(x)$ of the fermion operator as a function of its corresponding eigenvalue $\lambda_{n}$ for $L=8$ at $G=5.0$. The symmetry in the plot just reflects the fact that the fermion eigenvalues come in equal and opposite pairs as expected for an antisymmetric matrix. Clearly the low lying eigenstates have odd parity $\epsilon(x) \sim-1$ while the states with large eigenvalue have even parity $\epsilon(x) \sim+1$. This conclusion is reinforced if we look at the histogram of the values of $\epsilon(x)$ shown in Fig. 4 which shows that the distribution of values of $\langle n|\epsilon(x)| n\rangle$ cluster increasingly around \pm 1 as $G$ increases. As $G \rightarrow 0$ it is easy to verify that $\langle n|\epsilon(x)| n\rangle=0$ for all modes i.e., the double peaks merge back into a single peak at the origin. This is to be expected since $\phi_{n}(x)$ and $\epsilon(x) \phi_{n}(x)$ are orthogonal eigenvectors of the fermion matrix at $G=0$ which follows from the fact that $\epsilon$ anticommutes with the staggered fermion operator.

It is instructive to also examine the lowest eigenvalues of the fermion operator $M$ as the coupling $G$ is varied. This is shown in Fig. 5 which plots the lowest 32 eigenvalues of $M$ for three different values of the coupling $G$ on a $8^{2}$ lattice with periodic boundary conditions and $g=0.01$. Each

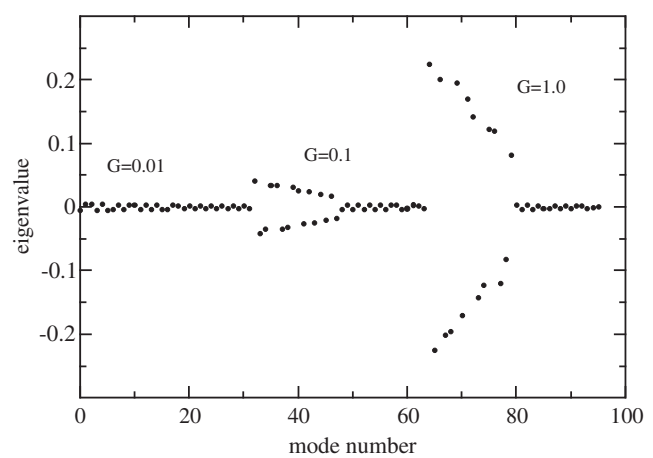

FIG. 5. First 32 eigenvalues of the fermion operator for $G=0.01, \quad G=0.1 \quad$ and $\quad G=1.0 \quad$ for $\quad 8^{2} \quad$ lattice at $g=0.01$ and pbc. 


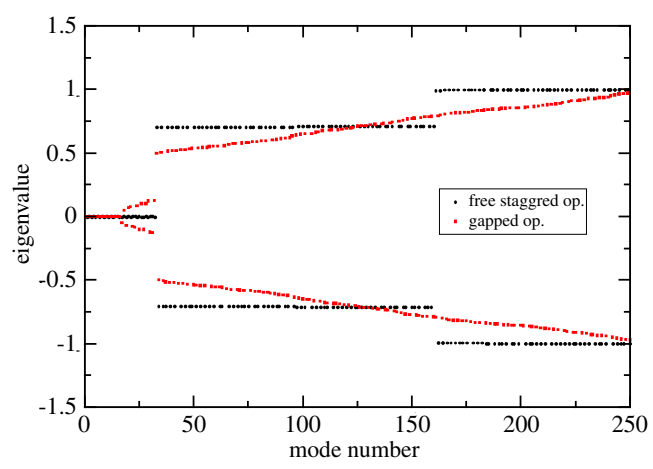

FIG. 6. Spectrum of $8^{2}$ periodic lattice at $g=0.001$ and $G=0.5$.

eigenvalue is given a unique label in the range 0-95 with the first 32 eigenvalues corresponding to $G=0.01$, the next 32 to $G=0.1$ and the final 32 to $G=1.0$. Notice again that the eigenvalues come in $\lambda,-\lambda$ pairs as expected from antisymmetry of $M$. At the smallest coupling we see that all 32 eigenvalues lie close to zero. This is to be expected since eight flavors of two dimensional massless real staggered fermions should have $4 \times 8=32$ exact zeroes. However as $G$ is increases half of these would be zero modes are progressively lifted away from the origin because of the strong Yukawa interaction. Indeed if we are successful at gapping half of the modes we would expect only sixteen would-be zero modes to remain corresponding to eight Weyl fermions in two dimensions. This is exactly what we see happens in this plot as $G$ increases.

Of course we are most interested in whether the low lying non-zero eigenvalues of the gapped theory correspond to what one would expect for a Weyl fermion. Figure 6 shows the lowest 256 eigenvalues of $M$ at $G=0.5$ and $g=0.001$ for an $8^{2}$ lattice with periodic boundary conditions. For comparison we also plot the spectrum of the free reduced staggered fermion operator for the same lattice. While we see a rough matching of the spectrum to that of a free fermion the correspondence is not perfect which presumably reflects the effects of the fluctuating scalar field which has a small but nonzero coupling to the odd parity fermions. However, in any case, one should be careful in reading too much from this comparison; the continuum Weyl fields are to be constructed from the odd parity staggered fields in each elementary square of the lattice and hence reside on a lattice with twice the original lattice spacing. It is the spectrum of the effective quadratic operator coupling these odd parity fields on this coarser lattice that is of primary interest. Construction of the effective quadratic operator governing these Weyl fermions on the coarser lattice is a future goal of our work and will be necessary to be sure the theory flows to a chiral theory in the continuum.

The other key issue we would like to understand better is whether the system develops a fermion bilinear condensate.
If it does the entire approach will fail. To see this go back to the form of the action written in Eq. (25) and replace $\sigma_{A}$ by a constant. Operating with $\eta_{\mu} D_{\mu}$ on the equation of motion for the light field yields

$$
D_{\mu}^{2} \chi_{-}-\sigma^{2} \chi_{-}=0
$$

which shows that the $\chi_{-}$field picks up a $\mu$ independent mass which is equal to that for the $\chi_{+}$field and indicates that the continuum limit will correspond to a Dirac fermion.

To test for this we have focused our attention on two particular bilinear operators - the site operator

$$
O_{1}=m_{0}\left\langle\chi^{T} \Gamma_{0} \chi\right\rangle
$$

and the link operator $[34,35]$

$$
O_{2}=m_{1} \sum_{\mu} \chi^{T}(x) \chi(x+\mu) \epsilon(x) \xi_{\mu}(x) .
$$

A vev for the former would spontaneously break $\operatorname{Spin}(7)$ and the $Z_{4}$ symmetry while a vev for the latter would explicitly couple the even and odd sectors. To test for these scenarios we have added explicit sources to the action and measured the vevs for several lattice volumes as the sources are sent to zero. In systems exhibiting spontaneous symmetry the corresponding vev develops a strong volume dependence as the source is sent to zero allowing for a nonzero vev to survive in the thermodynamic limit. However, as is clear in Fig. 7 and Fig. 8 which employ $G=5.0$ and $g=0.01$ no such strong volume dependence is seen for either bilinear and the vevs go smoothly to zero with vanishing external source. We thus conclude that there is no evidence for the formation of nonvanishing fermion bilinears at least in the two dimensional model.

It is perhaps useful to pause at the point to reiterate the basic strategy of this approach. In domain wall fermion approaches to chiral fermions one is able to separate the chiral modes in an extra fifth dimension. Here, in contrast, one uses Yukawa interactions to separate the eigenstates of

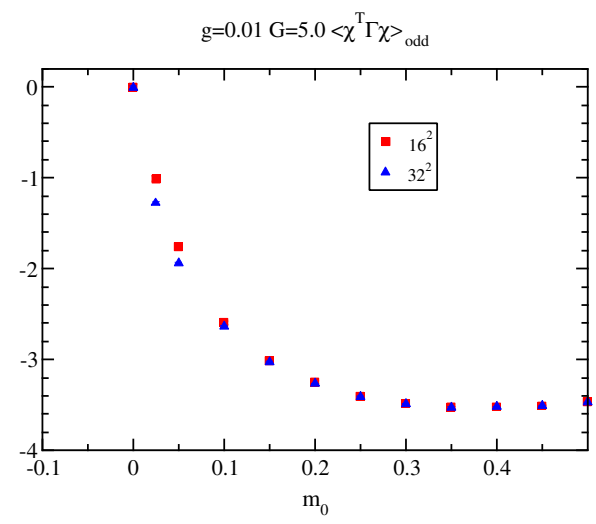

FIG. 7. $\left\langle\left(\chi^{T} \Gamma_{0} \chi\right)\right\rangle$ vs $m_{0}$ for $L=16$ and $L=32$. 
$\mathrm{g}=0.01 \mathrm{G}=5.0<\Sigma_{\mu} \chi(\mathrm{x}) \chi(\mathrm{xti} \mu)>$

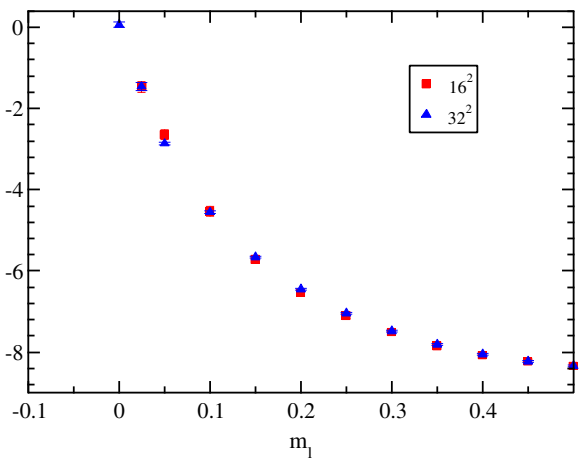

FIG. 8. $\left.\quad \sum_{\mu}\left\langle\chi^{T}(x) \chi(x+\mu) \epsilon(x) \xi_{\mu}(x)\right)\right\rangle$ vs $m_{1}$ for $L=16$ and $L=32$.

the parity operator $\epsilon(x)$ according to their eigenvalue (essentially separating them in momentum space). Of course for the lattice theory to target a chiral theory in the continuum limit requires that there be a correlation between $\epsilon(x)$ and $\gamma_{5}$. For regular staggered fermions containing both $\chi$ and $\bar{\chi}$ at each site there is no simple correspondence between the site parity operator $\epsilon(x)$ and the continuum chirality operator $\gamma_{5}$-lattice fields with a given value of $\epsilon(x)$ give rise to both left and right-handed continuum fermions. However, the correspondence becomes closer if one restricts to real staggered fields where the discussion in Sec. IV shows that $\epsilon(x)$ becomes a proxy for $\gamma_{5}$ at nonzero lattice spacing. However, even in that case, the continuum limit of the noninteracting theory is still vectorlike since it contains equal numbers of even and odd lattice fields leading to equal numbers of left and right-handed continuum fermions. If we now switch on $G$ it is no longer true that $\epsilon(x)$ is a proxy for $\gamma_{5}$ for the even parity fields. But it continues to be true for the odd parity fields since they are not subject to the Yukawa interaction. If symmetric mass generation occurs and the even parity modes are removed from the low energy spectrum and given a mass of order the cutoff we will be left with a theory that targets fermions of fixed chirality in the continuum limit. This is the strategy being pursued in this paper.

\section{SUMMARY AND PROSPECTS}

In this paper we have described a new lattice fermion mirror model which employs reduced staggered fermions transforming in the real eight dimensional chiral representation of a Spin(7) symmetry group. Interactions are introduced via Yukawa interactions of Fidkowski-Kitaev type on even parity lattice sites which we argue are capable of generating mass for half of the lattice fermions without producing symmetry breaking bilinear fermion condensates. Gapping fermions without breaking symmetries is called symmetric mass generation.

If these Yukawa couplings are able to generate cutoff scale masses for the even parity modes we argue that the remaining odd parity modes can be reorganized, in four dimensions, into sixteen free Weyl fermions in the continuum limit. This number of Weyl fermions is precisely what is needed to cancel off a discrete spin- $Z_{4}$ anomaly in the continuum theory and indeed the vanishing of this anomaly is a necessary condition to decouple the mirror fermions via symmetric mass generation. Indeed, with hindsight, the failure to cancel off these discrete anomalies was one of the primary reasons that many early efforts to construct lattice mirror models failed. In the appendix we give an argument that this fermion counting is also consistent with the cancellation of a novel gravitational anomaly that is unique to a generalization of staggered fermions propagating on lattices of arbitrary topology.

We show numerical evidence in favor of this scenario from simulations in two dimensions. The structure of this interaction, the arguments for symmetric mass generation, and the chiral properties of the continuum limit are identical to the case of four dimensions. In two dimensions a reduced staggered field contains four real degrees of freedom in a unit cell and hence yields a single Dirac fermion in the continuum limit. Since the model contains eight copies of this field the continuum limit of the free theory can be rewritten in terms of eight left and eight right-handed Weyl fermions. If symmetric mass generation occurs and Lorentz invariance is restored, the gapped theory will possess just eight left Weyl fermions in the continuum limit - a number which matches that required for cancellation of another discrete anomaly arising from chiral fermion parity $[17,18,22,38]$. For large Yukawa coupling $G$ our simulations shows that the low lying modes of the fermion operator do indeed have odd parity while the even parity modes are heavy. Furthermore, we observe that the number of zero modes of the fermion operator agrees with what is expected for 8 copies of a free Weyl fermion. Assuming Lorentz invariance is restored these ingredients suggest that free Weyl fermions will indeed be recovered in the continuum limit.

It also seems likely that a similar construction may work in three dimensions. In this case the unit cube on the lattice contains eight real staggered fields. Gapping say the even site parity fields would leave four light fields which can be assembled into two Majorana fermions in the continuum limit. Since these transform in an eight dimensional representation the continuum limit would then contain sixteen Majorana fermions again consistent with the expectation from continuum time reversal invariance.

In this paper the Spin(7) symmetry is a global symmetry. However, it can be straightforwardly gauged to yield a chiral lattice gauge theory in the continuum limit. One merely replaces the symmetric difference operator by the appropriate gauge covariant difference operator acting on fermions in the eight dimensional representation of $\operatorname{Spin}(7)$.

$D_{\operatorname{cov}} \chi(x)=\frac{1}{2}\left(U_{\mu}(x) \chi(x+\mu)-U^{\dagger}(x-\mu) \chi(x-\mu)\right)$ 
where $U_{\mu}(x)=e^{i \omega_{A B}\left[\Gamma_{A}, \Gamma_{B}\right]}$ are the gauge links for the real eight dimensional chiral representation of $\operatorname{Spin}(7)$. The interaction terms being purely local are already $\operatorname{Spin}(7)$ gauge invariant.

Of course, the question arises as to how one should take the continuum limit. Naively one would expect to have to search for a continuous phase transition in the theory as was done for the vectorlike case. However, while such a phase transition would presumably be needed to take a continuum limit for the strongly interacting sector, it does not appear necessary if the only fields of interest lie in the noninteracting odd parity sector. Indeed it appears that keeping $x=g G \ll 1$ fixed while $y=\frac{g}{G} \rightarrow 0$ may be sufficient. Since $G$ is naively an irrelevant coupling in four dimensions with mass dimension minus one could imagine scaling its bare coupling $G \sim L$ as $L \rightarrow \infty$ to retain a cutoff scale four fermion condensate for the even parity fields as the lattice size is increased. To keep $x$ fixed one would then simultaneously scale $g \sim \frac{1}{L}$. We are currently exploring this limit in more detail.

Some caveats are in order. We have assumed Lorentz invariance is restored in the continuum limit in order to identify the chirality and flavor representation of the fermions arising from the underlying staggered lattice fields. More specifically we have assumed that the low lying lattice modes organize themselves into Weyl spinors as the lattice spacing approaches zero. While this is consistent with our counting of degrees of freedom and the constraints from discrete anomalies it is a stronger requirement than simply the restoration of rotational invariance. It should be checked in future numerical work. In addition, while we have observed no sign problems for the simulations reported in this paper a sign problem will likely return once one gauges the Spin(7) flavor symmetry and/or takes the continuum limit. Finally, while we have see no evidence for the formation of off-site fermion bilinear condensates in two dimensions, this must be carefully checked in four dimensions.

\section{ACKNOWLEDGMENTS}

This work was supported by the U.S. Department of Energy (DOE), Office of Science, Office of High Energy Physics under Award No. DE-SC0009998. The author would like to thank Nouman Butt and Goksu can Toga for discussions and David Tong for patiently educating me about discrete anomalies.

\section{APPENDIX A: ANOMALIES AND STAGGERED FERMIONS}

There is another way of understanding why the lattice theories studied in this paper are subject to strong constraints on their fermion content. To exhibit these constraints it is necessary to generalize staggered fermions to lattices with non trivial topology. The starting point is to recognize that staggered fermions can be thought of as a particular discretization of Kähler-Dirac fermions-albeit one suitable only for regular toroidal lattices [39].

The Kähler-Dirac equation offers an alternative to the Dirac equation for curved spaces and reduces to the latter for flat spaces where it describes multiples of Dirac fermions (four flavors of Dirac fermion in four dimensions). It takes the form

$$
\left(d-d^{\dagger}+m\right) \Omega=0
$$

where $\Omega$ is a collection of antisymmetric tensor fields over the space and $d, d^{\dagger}$ are the exterior derivative and its adjoint. In flat space the tensor fields are given in terms of the spinor components of this set of Dirac fermions and the antisymmetry of the forms reflects the antisymmetric properties of the Clifford algebra associated to the Dirac gamma matrices.

A general procedure for discretizing the Kähler-Dirac equation on simplicial lattices was given in [40]. First, the antisymmetric component fields in $\Omega$ are placed on $\mathrm{p}$ simplices in an (oriented) triangulation of the space. Thus lattice fields are placed on sites (0-simplices), links (1simplices), triangles (2-simplices) etc. Next, a discrete boundary operator $\partial$ is defined whose action on some $p$-simplex decomposes it into an oriented list of its $(p-1)$ simplex boundary components. It can hence be used to create a map between $p$-simplex fields and $(p-1)$-simplex fields. In fact this boundary operator is the discrete analog of the adjoint of the exterior derivative. A similar lattice operator - the co-boundary operator $\bar{\partial}$ when acting on a $p$ simplex returns an oriented list of $(p+1)$-simplices that contain that $p$-simplex in their boundary. The coboundary operator replaces the exterior derivative and yields a map between $p$-simplex fields and $(p+1)$-simplex fields. The massless discrete Kähler-Dirac equation then takes the form

$$
(\partial-\bar{\partial}) \Omega=0 .
$$

Solutions of this equation go smoothly over into their continuum cousins as the lattice spacing is reduced-there is no additional fermion doubling.

In [41] it was shown that the corresponding Kähler-Dirac action - both continuum and discrete-is invariant under a $U(1)$ symmetry which distinguishes tensor fields with an even or odd number of indices. It acts as

$$
\begin{aligned}
& \Omega \rightarrow e^{i \alpha \Gamma} \Omega \\
& \bar{\Omega} \rightarrow \bar{\Omega} e^{i \alpha \Gamma}
\end{aligned}
$$

where $\Gamma$ anticommutes with the lattice Kähler-Dirac operator and takes the values \pm 1 on even and odd forms. This transformation is the analog of $e^{i \epsilon(x) \alpha}$ for staggered 
fermions on a torus but works on an arbitrary random triangulation of any topology. However, as was shown in [41] this symmetry is anomalous with the resulting partition function transforming by an overall phase $e^{i \alpha \chi}$ depending only on the Euler character of the triangulation which is given by

$$
\chi=N_{0}-N_{1}+\ldots(-1)^{p} N_{p}+\cdots+(-1)^{D} N_{D}
$$

Notice that this result holds equally well in the continuum for Kähler-Dirac fermions and shows that the anomaly can be computed exactly in the lattice theory since it depends only on the topology of the background space which can be captured exactly by the lattice. It is an example of an anomaly which does not require the presence of an infinite number of degrees of freedom.

Applying this result to the sphere $S^{2 n}$, which we can think of as representing a compactification of $R^{2 n}$, one finds that this $U(1)$ symmetry is broken to $Z_{4}$ in even dimensions. Thus, while a fermion bilinear operator is prohibited by this symmetry, it is possible to introduce four fermion operators. If want to retain Lorentz invariance we are forced to consider theories with at least four flavors of KählerDirac field. If we restrict to real representations these four complex Kähler-Dirac field can be decomposed into eight real Kähler-Dirac fields which are equivalent to eight reduced staggered fermions on a torus. This is precisely the field content we argued was necessary to formulate a mirror model capable of symmetric mass generation and yields a theory after gapping with exactly eight and sixteen massless Majorana fermions in two and four dimensions respectively. This suggests that there is a connection between the spin- $Z_{4}$ symmetry of the continuum and this $Z_{4}$ symmetry of Kähler-Dirac fermions.

\section{APPENDIX B: NUMERICAL METHODS}

If we integrate out the fermions we obtain a Pfaffian $\operatorname{Pf}(M)$. Provided this is positive definite we can replace it by $\operatorname{det}\left(M^{\dagger} M\right)^{\frac{1}{4}}$. In practice the latter is generated by integrating over a set of commuting pseudofermion fields with action

$$
S_{\mathrm{PF}}=\phi^{T}\left(M^{\dagger} M\right)^{-\frac{1}{4}} \phi=\sum_{i}^{N} \phi^{T}\left[\frac{\alpha_{i}}{M^{\dagger} M+\beta_{i}}\right] \phi
$$

where $\phi^{a}(x)$ is vector indexed by the lattice sites and Spin(7) label. The second expression represents a rational fraction approximation to the fractional power of the matrix. The coefficients $\alpha_{i}$ and $\beta_{i}$ are determined for a given number of terms $N$ by the remez algorithm - see [42]. In practice we set $N=18$ and tolerate a relative error of $10^{-8}$ over the eigenvalue interval 0.00000001-1000.0. We use a multi-timestep Omelyan integrator to generate an auxiliary classical dynamics that is used to sample the partition function of the system where the configurations are subject to a Metropolis test after each molecular dynamics trajectory. The time consuming part of this evolution corresponds to the calculation of the force terms arising from the pseudofermion action, For these we use a multi-shift CG solver. We have implemented a parallelized version of this algorithm by using the MILC communication libraries and the code runs efficiently on clusters with dedicated networking.

Our results derive from ensembles of 2000 configurations for a given lattice size and set of couplings with measurements taken every 10 sweeps. Errors are assessed as usual by a jackknife procedure and a set of $Z_{2}$ stochastic sources are used to estimate condensates.
[1] H. B. Nielsen and M. Ninomiya, Phys. Lett. 105B, 219 (1981).

[2] D. M. Grabowska and D. B. Kaplan, Phys. Rev. Lett. 116, 211602 (2016).

[3] E. Eichten and J. Preskill, Nucl. Phys. B268, 179 (1986).

[4] W. Bock, J. Smit, and J. C. Vink, Nucl. Phys. B414, 73 (1994).

[5] W. Bock and A. K. De, Phys. Lett. B 245, 207 (1990).

[6] A. Hasenfratz, P. Hasenfratz, K. Jansen, J. Kuti, and Y. Shen, Nucl. Phys. B365, 79 (1991).

[7] A. Hasenfratz and T. Neuhaus, Phys. Lett. B 220, 435 (1989).

[8] I.-H. Lee, J. Shigemitsu, and R. E. Shrock, Nucl. Phys. B330, 225 (1990).

[9] M. F. Golterman, D. N. Petcher, and E. Rivas, Nucl. Phys. B395, 596 (1993).
[10] E. Poppitz and Y. Shang, J. High Energy Phys. 03 (2009) 103.

[11] E. Poppitz and Y. Shang, Int. J. Mod. Phys. A 25, 2761 (2010).

[12] C. Chen, J. Giedt, and E. Poppitz, J. High Energy Phys. 04 (2013) 131.

[13] Y. Kikukawa, Prog. Theor. Exp. Phys. (2019), 113 B03.

[14] M. Creutz, M. Tytgat, C. Rebbi, and S.-S. Xue, Phys. Lett. B 402, 341 (1997).

[15] M. Luscher, Nucl. Phys. B549, 295 (1999).

[16] L. Fidkowski and A. Kitaev, Phys. Rev. B 81, 134509 (2010).

[17] S. Ryu and S.-C. Zhang, Phys. Rev. B 85, 245132 (2012).

[18] X.-L. Qi, New J. Phys. 15, 065002 (2013).

[19] Y.-Z. You and C. Xu, Phys. Rev. B 91, 125147 (2015).

[20] Y. Tachikawa and K. Yonekura, SciPost Phys. 7, 058 (2019). 
[21] A. Kapustin, R. Thorngren, A. Turzillo, and Z. Wang, J. High Energy Phys. 12 (2015) 052.

[22] I. n. García-Etxebarria and M. Montero, J. High Energy Phys. 08 (2019) 003.

[23] V. Ayyar and S. Chandrasekharan, Phys. Rev. D 91, 065035 (2015).

[24] V. Ayyar and S. Chandrasekharan, Phys. Rev. D 93, 081701 (2016).

[25] S. Catterall, J. High Energy Phys. 01 (2016) 121.

[26] S. Catterall and D. Schaich, Phys. Rev. D 96, 034506 (2017).

[27] N. Butt, S. Catterall, and D. Schaich, Phys. Rev. D 98, 114514 (2018).

[28] V. Ayyar and S. Chandrasekharan, J. High Energy Phys. 10 (2016) 058.

[29] V. Ayyar and S. Chandrasekharan, Phys. Rev. D 96, 114506 (2017).

[30] Y.-Z. You, Y.-C. He, C. Xu, and A. Vishwanath, Phys. Rev. X 8, 011026 (2018).

[31] Y. You, Y. BenTov, and C. Xu, arXiv:1402.4151.
[32] J. Wang and X.-G. Wen, Phys. Rev. Research 2, 023356 (2020).

[33] J. Wang and X.-G. Wen, Phys. Rev. D 99, 111501 (2019).

[34] M. F. Golterman and J. Smit, Nucl. Phys. B245, 61 (1984).

[35] C. van den Doel and J. Smit, Nucl. Phys. B228, 122 (1983).

[36] W. Bock, J. Smit, and J. C. Vink, Phys. Lett. B 291, 297 (1992).

[37] S. Catterall, D. B. Kaplan, and M. Unsal, Phys. Rep. 484, 71 (2009).

[38] S. S. Razamat and D. Tong, Phys. Rev. X 11, 011063 (2021).

[39] T. Banks, Y. Dothan, and D. Horn, Phys. Lett. 117B, 413 (1982).

[40] J. M. Rabin, Nucl. Phys. B201, 315 (1982).

[41] S. Catterall, J. Laiho, and J. Unmuth-Yockey, J. High Energy Phys. 10 (2018) 013.

[42] M. A. Clark, A. D. Kennedy, and Z. Sroczynski, Nucl. Phys. B, Proc. Suppl. 140, 835 (2005). 